The Astrophysical Journal, 669:606-614, 2007 November 1

(C) 2007. The American Astronomical Society. All rights reserved. Printed in U.S.A.

\title{
A DECREASED PROBABILITY OF HABITABLE PLANET FORMATION AROUND LOW-MASS STARS
}

\author{
Sean N. Raymond, ${ }^{1,2}$ John Scalo, ${ }^{2,3}$ and Victoria S. Meadows ${ }^{2,4}$ \\ Received 2006 March 2; accepted 2007 July 11
}

\begin{abstract}
Smaller terrestrial planets $\left(\lesssim 0.3 M_{\oplus}\right)$ are less likely to retain the substantial atmospheres and ongoing tectonic activity probably required to support life. A key element in determining whether sufficiently massive sustainably habitable planets can form is the availability of solid planet-forming material. We use dynamical simulations of terrestrial planet formation from planetary embryos and simple scaling arguments to explore the implications of correlations between terrestrial planet mass, disk mass, and the mass of the parent star. We assume that the protoplanetary disk mass scales with stellar mass as $M_{\text {disk }} \propto f M_{\star}^{h}$, where $f$ measures the relative disk mass and $1 / 2<h<2$, so that disk mass decreases with decreasing stellar mass. We consider systems without Jovian planets, based on current models and observations for $\mathrm{M}$ stars. We assume the mass of a planet formed in some annulus of a disk with given parameters is proportional to the disk mass in that annulus and show with a suite of simulations of late-stage accretion that the adopted prescription is surprisingly accurate. Our results suggest that the fraction of systems with sufficient disk mass to form $>0.3 M_{\oplus}$ habitable planets decreases for low-mass stars for every realistic combination of parameters. This habitable fraction is small for stellar masses below a mass in the interval $0.5-0.8 M_{\odot}$, depending on disk parameters, an interval that excludes most M stars. Radial mixing and therefore water delivery are inefficient in the lower mass disks commonly found around low-mass stars, such that terrestrial planets in the habitable zones of most low-mass stars are likely to be small and dry.
\end{abstract}

Subject headings: astrobiology — planetary systems: formation — planetary systems: protoplanetary disks — stars: low-mass, brown dwarfs

\section{INTRODUCTION}

The circumstellar habitable zone (HZ) is the distance annulus within which a terrestrial planet can maintain liquid water on its surface. The average distance of the HZ from the parent star depends primarily on the star's luminosity and is closer in and narrower around lower mass stars (Kasting et al. 1993). For a planet to remain habitable, it must control its surface temperature over long timescales, possibly via the $\mathrm{CO}_{2}$-carbonate cycle (Walker et al. 1981), which is enabled by a relatively thick atmosphere, a hydrological cycle, and active plate tectonics. These planetary characteristics are less likely to be sustained over long periods by less massive terrestrial planets or those with low water contents. Whether potentially habitable planets of sufficient mass will form depends on the surface density of rocky material in their parent protoplanetary disks. Disks with higher surface densities tend to form a smaller number of more massive planets (Wetherill 1996; Raymond et al. 2004; Kokubo et al. 2006) and can increase terrestrial planet water content by scattering more distant, water-rich material into the HZ (Morbidelli et al. 2000; Raymond et al. 2004, 2005a).

Here we investigate the formation, masses, and water contents of terrestrial planets as a function of stellar mass by assuming a parameterized relationship between disk and stellar mass. We adopt a simple model relating the HZ planet mass to the disk mass, based on results in the literature. We use a set of numerical simulations of terrestrial planet growth around stars between 0.2 and

\footnotetext{
1 Center for Astrophysics and Space Astronomy, University of Colorado, 389 UCB, Boulder CO 80309-0389; raymond@lasp.colorado.edu. NASA Postdoctoral Program Fellow.

2 Member of NASA Astrobiology Institute.

3 Department of Astronomy, University of Texas, Austin, TX; parrot@as .utexas.edu.

4 Infrared Processing and Analysis Center, California Institute of Technology, Pasadena, CA; vsm@ipac.caltech.edu.
}

$1 M_{\odot}$ to test the validity of the model. The simulations agree well with the model and justify an exploration of the consequences of such a planet disk-stellar mass relation, and in addition allow us to consider how water delivery is affected as a function of stellar mass.

Our main result is to derive probabilistic limits on the stellar mass below which sustainably habitable planets cannot form because of insufficient disk mass available for accretion onto a critical planet mass of $0.3 M_{\oplus}$. In addition, we show how this stellar mass limit depends on the parameters of the disk-star mass relationship, which is currently poorly constrained. In a statistical framework, we show that the fraction of stars capable of forming habitable planets in situ decreases quickly with decreasing stellar mass and that terrestrial planets in the habitable zones of $\mathrm{M}$ stars $\left(M_{\star} \lesssim 0.5 M_{\odot}\right.$; e.g., Reid \& Hawley 2000$)$ are likely to be small and dry. An interesting test of our models comes from the recent detection of a $\sim 5 M_{\oplus}$ planet close to the habitable zone of the M dwarf Gliese 581 (Udry et al. 2007). The implications of this system for our formation models are discussed in $\S 4$.

To simplify our calculations, we assume there are no gas giant (Jovian) planets present, and we test the effects of this assumption in $\S 3.3$. Systems without giant planets may represent the majority of planetary systems with habitable planets. Available estimates of the fraction of solar-like stars with massive planets (Tabachnik \& Tremaine 2002; Lineweaver \& Grether 2003; Beer et al. 2004; Fischer \& Valenti 2005) allow for 70\%-90\% of stars to have planetary systems without Jovians, and an even larger fraction for lower mass stars (Endl et al. 2006; Butler et al. 2006). In addition, Scholz et al. (2006) point out that estimated submillimeter and millimeter outer disk masses show that only a small fraction of low-mass stars or substellar objects have sufficient disk mass to form a planet with the mass of Jupiter (but see Hartmann et al. 2006). The lack of a correlation between the presence of debris disks and the metallicity of the parent star (Greaves et al. 2006; Beichman et al. 2006; Moro-Martín et al. 2007), unlike the 
correlation known for exogiant planets (Gonzalez 1997; Santos et al. 2001; Fischer \& Valenti 2005), also supports the likelihood of gas-giant-free planetary systems. There are observational (Endl et al. 2006; Butler et al. 2006; Gould et al. 2006) and theoretical (Ward \& Hahn 1995; Adams et al. 2004; Laughlin et al. 2004; Ida $\&$ Lin 2005) reasons to think that the fraction of M stars with Jovian planets is significantly smaller than for solar-mass stars.

\section{METHODS}

\subsection{Habitable Planet Mass Limit}

Following Williams et al. (1997), we assume a lower mass limit for planetary habitability that supports active plate tectonics for several Gyr. Significant mass is a necessary, but not sufficient, requirement for sustainable planetary habitability (e.g., Lissauer 1999). Using the radioactive flux limit from Williams et al. (1997), the critical planetary mass scales as $\rho^{-2} e^{3 \lambda t}$, where $\rho$ is the planet's bulk density, $\lambda=1.5 \times 10^{10} \mathrm{yr}^{-1}$ for ${ }^{238} \mathrm{U}$, and $t$ is the duration of tectonic activity. For an active lifetime of $5 \mathrm{Gyr}$, iterating on the density to be consistent with the resulting critical mass, we get a critical mass of $0.3 M_{\oplus}$ for a density of $4.5 \mathrm{~g} \mathrm{~cm}^{-3}$. This result is sensitive to the plate tectonic activity timescale associated with sustained habitability, which is fairly arbitrary. The average age of stars in the Galactic disk is about 5 Gyr, so the choice of 5 Gyr allows about half of such stars with sustainably habitable planets to be on the main sequence today, for a roughly constant star formation rate. Throughout the paper we consider $0.3 M_{\oplus}$ our critical mass limit for planetary habitability, and we discuss the implications of changing this limit in $\S 4$.

\subsection{Protoplanetary Disk Properties}

We assume the surface density of protoplanetary disks, $\Sigma$, to scale with heliocentric distance $r$ as

$$
\Sigma(r)=\Sigma_{1} f Z\left(\frac{r}{1 \mathrm{AU}}\right)^{-\alpha}\left(\frac{M_{\star}}{M_{\odot}}\right)^{h},
$$

where $\Sigma_{1}$ is the surface density of solids at 1 AU in the minimummass solar nebula (MMSN) model $\left(\approx 6 \mathrm{~g} \mathrm{~cm}^{-2}\right), h$ adjusts the density scaling with $M_{\star}, f$ is a scaling factor for the disk ( $f=1$ for MMSN $), Z$ is the stellar metallicity $(1.0=$ solar $)$, and $\alpha$ moderates the steepness of the density profile $(\alpha=3 / 2$ in the MMSN model; Weidenschilling 1977; Hayashi 1981; but more recent reconstructions using an accretion disk model as a constraint give $\alpha=1 / 2$; Davis 2005). Most disk models that include viscous and irradiation heating give $\alpha$ between about $1 / 2$ and 1 for the inner disk (e.g., D’Alessio et al. 1998; Garaud \& Lin 2007; Dullemond et al. 2007), and high-resolution submillimeter observations suggest $\alpha$ between $1 / 2$ and 1 in the outer disk (Mundy et al. 2000; Looney et al. 2003; Andrews \& Williams 2007). We take $\alpha=1$ as a fiducial value, but consider values in the range $1 / 2-3 / 2$.

Present estimates of disk masses in nearby star-forming regions (see Andre \& Montmerle 1994; Eisner \& Carpenter 2003; Andrews $\&$ Williams 2005) give a large spread, with median or average masses usually somewhat below the MMSN value, but a significant fraction more massive, including very massive disks observed in both Orion and Taurus (e.g., Williams et al. 2005; Eisner $\&$ Carpenter 2006). At the other end of the mass spectrum, the estimates by Williams et al. (2005) of mean masses for 18 Orion proplyds below the $3 \sigma$ detection limit yielded $8 \times 10^{-4} M_{\odot}$, or only $3 M_{\oplus}$ in solids, for a gas-to-dust ratio of 100 . There also exists a factor of 3-5 range in metallicity for thin-disk stars (Nordstrom et al. 2004), which adjusts the effective gas-to-dust ratio.
There is considerable uncertainty in the scaling between stellar mass $M_{\star}$ and disk mass $M_{\text {disk }}$. The most promising lead comes from estimates of outer disk masses using submillimeter and millimeter techniques (Beckwith et al. 1990), which remain uncertain because of adopted dust temperatures, opacities, distances, and radiative transfer model. Scholz et al. (2006) estimated disk masses for brown dwarf disks and combined their results with previous determinations for stellar and substellar objects from 0.02 to $3 M_{\odot}$. Their Figure 3 shows that the ratio of disk mass to stellar mass is roughly constant with stellar mass, with a large amount of scatter. This hints that $h \approx 1$, with a large range in $f$. Many of the data points from Scholz et al. (2006) are only upper limits, but we think extreme cases with $h=0$ or $h>2$ would be apparent in this data.

Additional clues come from the observed stellar accretion rate $\dot{M}$, which scales as $\dot{M} \propto M_{\star}^{2}$, with a large dispersion (Muzerolle et al. 2003, 2005; Natta et al. 2006). It might therefore seem natural to assume that $M_{\text {disk }} \propto M_{\star}^{2}$, i.e., $h=2$, assuming that the viscous timescale is independent of stellar mass (e.g., Ida \& Lin 2005). On the contrary, nearly all accretion disk models predict $\dot{M} \propto M_{\star}^{a}$ with $a \sim 1$. This matter is still unresolved; several solutions have been proposed (see Padoan et al. 2005; Alexander \& Armitage 2006; Dullemond et al. 2006; Hartmann et al. 2006; Gregory et al. 2006), including selection/detection limitations (Clarke \& Pringle 2006). Most of these models are consistent with $h \approx 1$. Although $h$ is currently poorly constrained, we emphasize its importance for understanding whether sustainable HZ planets can form around low-mass stars. Given the results of Scholz et al. (2006), we choose $h=1$ as our fiducial case, but explore the effects of $h$ from 0.5 to 2 .

\subsection{Simulations and Models}

We performed dynamical simulations to evaluate the scaling between terrestrial planet mass and disk properties. For all runs, our starting conditions reflect our fiducial case, with $f Z=1$, $h=1$, and $\alpha=1$. We vary the mass of the central star from 0.2 to $1 M_{\odot}$. Given the surface density profile from equation (1), we assume that planetary embryos have formed throughout the inner disk of each star. We assume that the disk evolved following the standard model of terrestrial planet growth (e.g., Chambers 2004): grains coalesced to form $\mathrm{km}$-sized planetesimals, and embryos formed from planetesimals via runaway and oligarchic growth (e.g., Kokubo \& Ida 1998), spaced by 5-10 mutual Hill radii $\left[R_{\mathrm{H}, \mathrm{m}}=0.5\left(a_{1}+a_{2}\right)\left(M_{1}+M_{2} / 3 M_{\star}\right)^{1 / 3}\right.$, where $a_{1}$ and $a_{2}$ are the orbital radii and $M_{1}$ and $M_{2}$ are the masses of two adjacent embryos]. For the case of $1 M_{\odot}$, we generated a population of $\sim 75$ embryos from 0.5 to $4 \mathrm{AU}$, totaling $4.95 M_{\oplus} \cdot{ }^{5}$ For other stellar masses, we assume that the population of embryos has the same temperature, as we are interested in $\mathrm{HZ}$ planets (defined to have the same temperature), and the feeding zone boundaries should scale in the same way as the HZ mean distance. We therefore scale the inner and outer boundaries by the stellar flux (i.e., as the luminosity $L_{\star}^{1 / 2}$ ), using a mass-luminosity relation from Scalo et al. (2007) that is a fit to data from Hillenbrand \& White (2004): $y=4.101 x^{3}+8.162 x^{2}+7.108 x+0.065$, where $y=\log L_{\star}$ and $x=\log M_{\star}$. Table 1 summarizes our starting conditions for each stellar mass. Note the variations in the number of embryos $N$ included in each simulation, from $\sim 75$ for the $1 M_{\odot}$ simulations to almost 200 for the $0.2 M_{\odot}$ simulations. This variation in $N$ is due to the Hill radius being very small at the small orbital distances studied for lower mass stars. In other words, models of

\footnotetext{
5 Note that the outer edge of 4 AU for the embryo disk is chosen based on previous simulations showing that the feeding zone of a terrestrial planet in the habitable zone does not extend beyond 4 AU (Raymond et al. 2007b).
} 
TABLE 1

Initial Conditions for Simulations

\begin{tabular}{ccccccc}
\hline \hline \\
$\begin{array}{c}M_{\star} \\
(1)\end{array}$ & $\begin{array}{c}\text { Range } \\
(\mathrm{AU}) \\
(2)\end{array}$ & $\begin{array}{c}\text { Mass } \\
\left(M_{\oplus}\right) \\
(3)\end{array}$ & $\begin{array}{c}N \\
(4)\end{array}$ & $\begin{array}{c}\text { Time Step } \\
(\text { days }) \\
(5)\end{array}$ & $\begin{array}{c}\mathrm{HZ} \\
(\mathrm{AU}) \\
(6)\end{array}$ & $\begin{array}{c}\text { Water Line } \\
(\mathrm{AU}) \\
(7)\end{array}$ \\
\hline $1.0 \ldots \ldots \ldots$. & $0.5-4$ & 4.95 & 75 & 6 & $0.8-1.5$ & 2.5 \\
$0.8 \ldots \ldots \ldots .$. & $0.25-2$ & 1.98 & 75 & 2.5 & $0.39-0.74$ & 1.23 \\
$0.6 \ldots \ldots \ldots .$. & $0.12-1$ & 0.75 & 100 & 1.0 & $0.20-0.37$ & 0.61 \\
$0.4 \ldots \ldots \ldots .$. & $0.06-1$ & 0.53 & 130 & 0.4 & $0.10-0.19$ & 0.32 \\
$0.2 \ldots \ldots \ldots .$. & $0.03-0.5$ & 0.13 & 190 & 0.2 & $0.05-0.1$ & 0.16 \\
\hline
\end{tabular}

Notes.-Col. (1): $M_{\star}$ is the stellar mass in solar masses. Col. (2): The range is the initial radial distribution of embryos. Col. (3): Mass represents the total mass in embryos. Col. (4): $N$ is the number of embryos. Col. (5): The time step is the time step used for the integration in days. Col. (6): HZ is the extent of the habitable zone for each case. Col. (7): The water line indicates the boundary beyond which embryos are assumed to contain $5 \%$ water by mass.

embryo growth predict that there really are more embryos in these inner disks than in the more distant regions studied for higher mass stars (e.g., Kokubo \& Ida 1998). Kokubo et al. (2006) showed that the bulk properties of accreted planets are not particularly sensitive to such variations in $N$, so we are not concerned that our choice of embryo formation models will affect our results. Note also that the $0.2 M_{\odot}$ star has less than $0.3 M_{\oplus}$ in its entire disk, so it serves primarily to tell us about the efficiency with which the disk mass is used to make planets under these conditions, but cannot form a habitable planet according to our criterion. Each numerical experiment was run independently four times starting from different random initial embryo positions for a total of 20 simulations. We give embryos random starting eccentricities $<0.02$ and inclinations $<0.1^{\circ}$.
Embryos are assigned water contents based on values from our solar system, where asteroids beyond $\sim 2.5 \mathrm{AU}$ contain significant quantities of water (Abe et al. 2000; see Fig. 2 in Raymond et al. 2004). We assumed that this boundary between "dry" and "wet" embryos (called the "water line" in Table 1) scales with the stellar flux. ${ }^{6}$ Embryos beyond this boundary contain 5\% water by mass, and those inside are dry (Table 1). We integrated each simulation for 200 Myr using the hybrid integrator Mercury (Chambers 1999). Time steps were chosen to sample the innermost body's orbit at least 20 times (e.g., Rauch \& Holman 1999; Levison \& Duncan 2000; see Table 1) and so varied with each set of simulations, from 6 days for $1 M_{\odot}$ to 0.2 days for $0.2 M_{\odot}$. Collisions were treated as inelastic mergers conserving water.

\section{RESULTS}

\subsection{Terrestrial Planet Mass versus Stellar Mass}

Figure 1 shows the evolution of a simulation for a $0.6 M_{\odot}$ star: the disk is excited by gravitational perturbations among the embryos. As eccentricities increase, orbits cross and collisions occur. In time, a few planets grow and the number of bodies dwindles. Embryos may be scattered far from their original locations, sometimes delivering water-rich material to planets in the inner regions. Water delivery occurs relatively late, because multiple scattering

\footnotetext{
${ }^{6}$ We use the term "water line" instead of the more common "snow line" because we are not necessarily assuming this to be the location where the temperature drops below $\sim 170 \mathrm{~K}$ and water ice can condense (2.7 AU in the MMSN; Hayashi 1981). Rather, we are simply assuming this boundary to divide dry and wet material. The water line may actually be located somewhat interior to the snow line, as water-rich bodies can be shifted inward either via gas drag or by eccentricity pumping during embryo formation (e.g., Cyr et al. 1998; Kokubo \& Ida 1998).
}

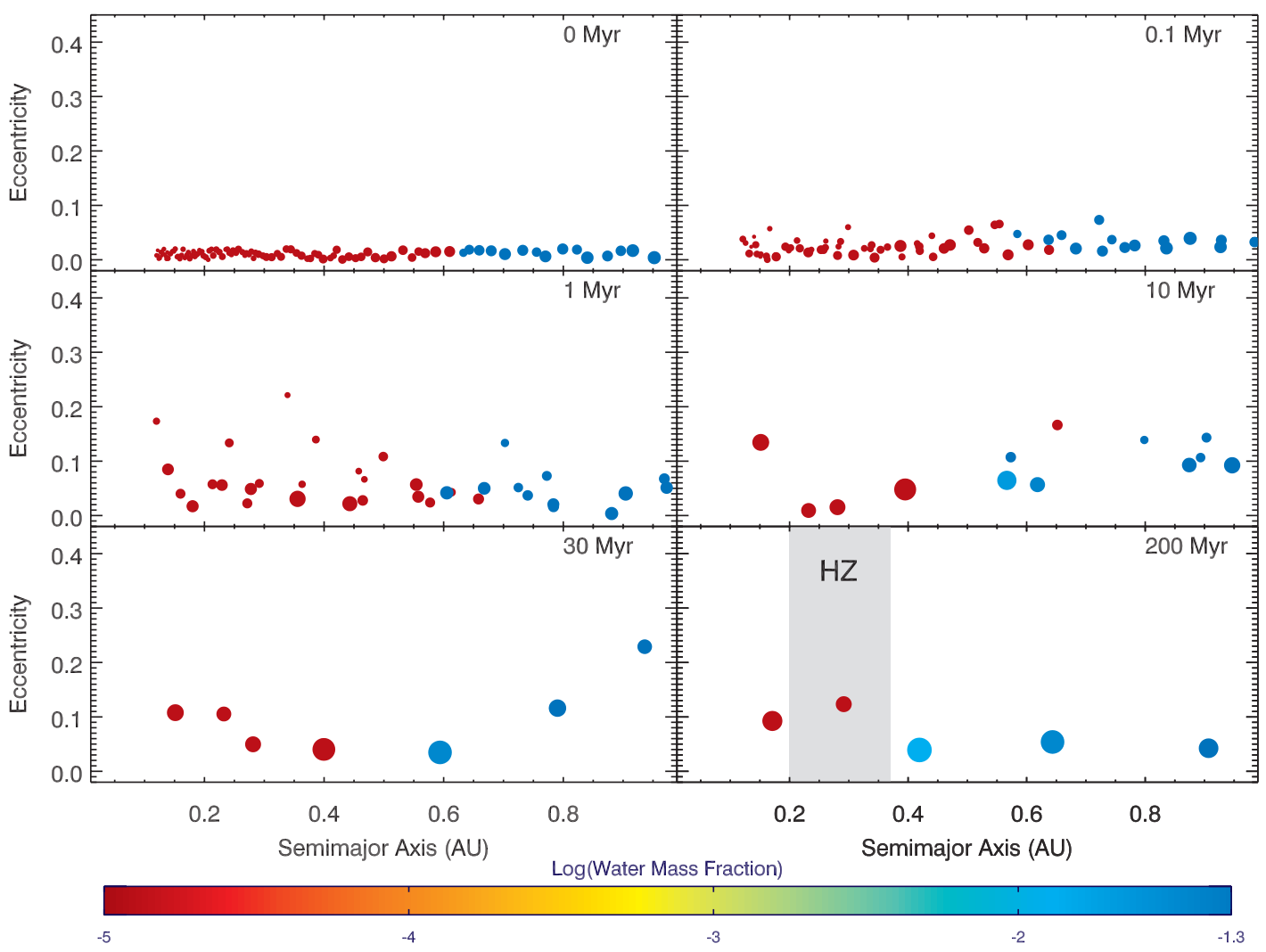

FIG. 1. - Evolution of a simulation around a $0.6 M_{\odot}$ star. Particle size scales with the planetary size as the (planet mass) ${ }^{1 / 3}$, but is not to scale on the $x$-axis. Colors correspond to water contents, from red (dry) to blue ( $5 \%$ water by mass; see color bar). For scale, the planet that formed at $0.29 \mathrm{AU}$ in the $\mathrm{HZ}$ is $0.06 M_{\oplus}$, and the planet at $0.42 \mathrm{AU}$ is $0.21 M_{\oplus}$. The $\mathrm{HZ}$ is shaded in the final panel of the simulation. Note that, although the HZ planet is dry, some water delivery did occur, as two water-rich embryos were accreted by the planet at $0.41 \mathrm{AU}$. 

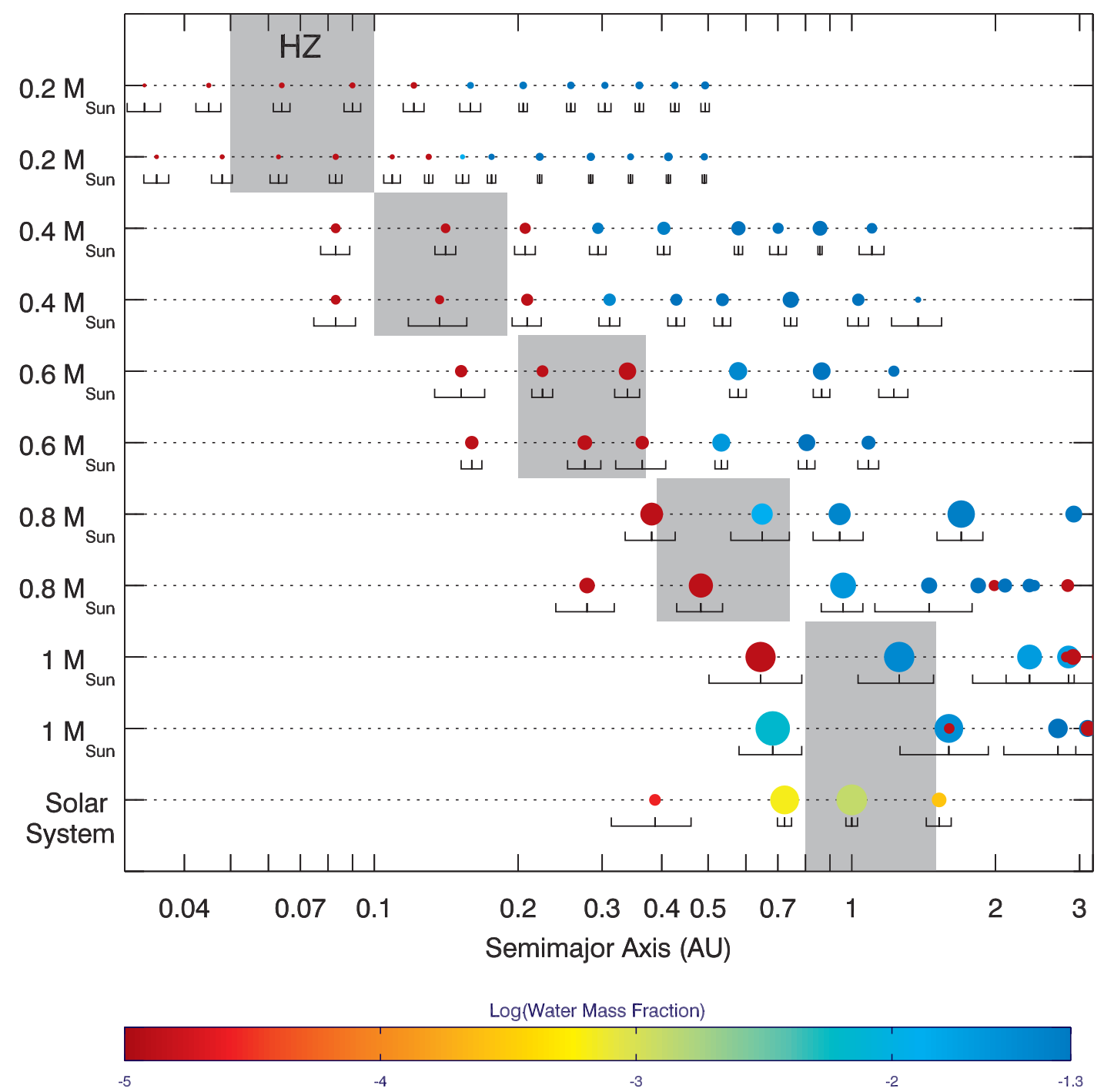

FIg. 2.-Final outcomes of 10 simulations, two for each stellar mass chosen, with the solar system for scale. As in Fig. 1, color represents water content, and particle size scales with the planet size, i.e., ( planet mass ${ }^{1 / 3}$ ). The lines under each planet represent the radial excursion of a planet over its orbit, i.e., its orbital eccentricity; these values are averaged over the last 10 Myr of each simulation. Solar system water contents are from Lodders \& Fegley (1998) and Lécuyer et al. (1998), and eccentricities are 3 Myr averages from Quinn et al. (1991). The HZ is shaded for each stellar mass.

events are needed for significant radial movement (Raymond et al. 2007b). In this case some water delivery occurred: a $0.21 M_{\oplus}$ planet formed at $0.41 \mathrm{AU}$, just beyond the outer boundary of the $\mathrm{HZ}$, accreting two water-rich embryos originating beyond the water line at $0.61 \mathrm{AU}$. However, no water was delivered to any planets in the $\mathrm{HZ}$ in this case. This is a typical outcome for the low-mass disks, expected to be common around low-mass stars (see below).

Figure 2 shows the final outcome of 10 simulations, with the solar system included for scale (the Earth's water content is $\sim 10^{-3}$ by mass; Lécuyer et al. 1998). It is clear that, for our assumptions of $h=1, \alpha=1$, and $h=1$, terrestrial planets are much smaller around low-mass stars. In addition, planets that form in the HZs of low-mass stars tend to be dry and more closely spaced. Note that, although we do not follow their orbits for a full 5 Gyr, we assume water-rich planets that form in the HZ to be potentially habitable. Very late stage instabilities were not seen in any of these simulations, nor in the $>$ Gyr integrations from Raymond et al. (2005a, $2006 \mathrm{~b}$ ), but the potential disruption of the system at times as late as 5 Gyr cannot be ruled out. In addition, a late-stage instability could alter the orbit of a distant planet, causing it to collide with a planet in the HZ. Such an event could even deliver water at a very late stage. Although this is certainly possible, we have found this type of event to be rare in previous long-term simulations. In addition, the source of such a rogue planet would have to be quite far out, because accretion tends to occur faster closer to the star, and crossing orbits are needed to cause a strong scattering event.

Figure 3 shows the mean mass of simulated planets that formed in the $\mathrm{HZ}$ as a function of stellar mass, with error bars representing the range of outcomes from the four simulations for a given stellar mass $M_{\star}$. The solid curve shows the prediction of a simple scenario in which the mass of a planet in the HZ is proportional to the mass contained within the $\mathrm{HZ}$ annulus, such that

$$
M_{\mathrm{pl}} \propto \frac{\Sigma_{1} f Z M_{\star}^{h}}{2-\alpha}\left(r_{\mathrm{HZ}, \text { out }}^{2-\alpha}-r_{\mathrm{HZ}, \text { in }}^{2-\alpha}\right),
$$

where $r_{\mathrm{HZ} \text {,in }}$ and $r_{\mathrm{HZ} \text {,out }}$ are the inner and outer boundaries of the HZ (see Table 1). This model is a very simple, but not unreasonable, approximation for planet mass, and the quality of the fit is remarkable - note that this model was calibrated such that a disk with $f Z=1$ will form a $1 M_{\oplus}$ planet in the $\mathrm{HZ}$ around a solarmass star. Kokubo et al. (2006) showed that the planet mass scales 


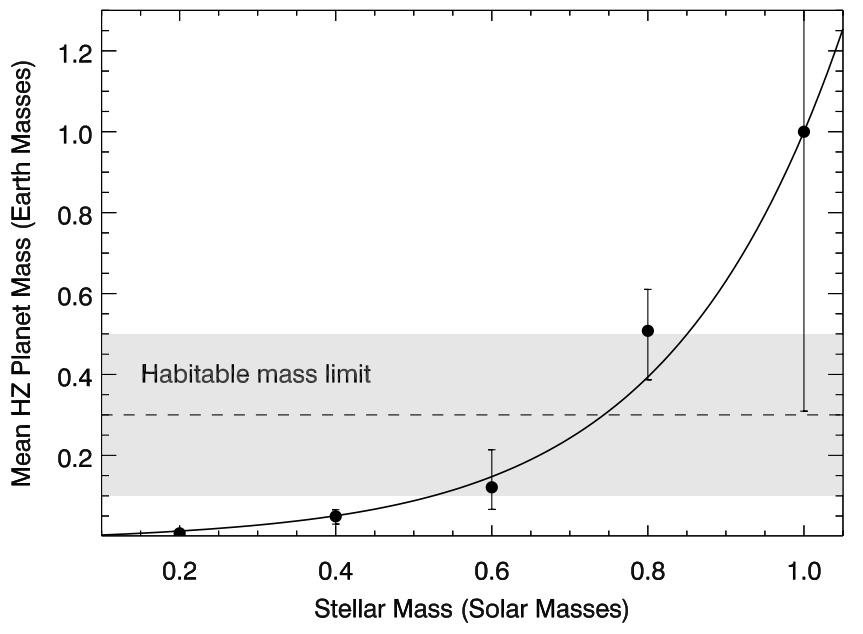

FIG. 3.- Mass of planets formed in the $\mathrm{HZ}$ as a function of stellar mass, for a model with $h=1, \alpha=1$, and $f Z=1.2$ (so that the mean planet mass for a $1 M_{\odot}$ star is $\left.1 M_{\oplus}\right)$. Error bars represent the range of values for $\mathrm{HZ}$ planets. The solid curve represents a model in which the HZ planet mass scales linearly with the total annular mass in the HZ. The shaded region represents reasonable estimates of the limiting planet mass for habitability $\left(0.1-0.5 M_{\oplus}\right)$; our chosen value of $0.3 M_{\oplus}$ is indicated by the dashed line.

roughly linearly with the available mass, albeit for a fixed stellar mass, and we independently found the same result for $M_{\star}=$ $0.4 M_{\odot}$. In fact, Kokubo et al. (2006) found a slightly stronger than linear correlation, $M_{\mathrm{pl}} \propto M_{\mathrm{disk}}^{0.97-1.1}$. Figure 2 shows that the mean interplanetary spacing decreases somewhat for lower mass stars and that there are slightly more planets for the lower mass stars. We know that the total mass in the habitable zone, $M_{\mathrm{HZ}}$, is equal to the number of planets, $N$, times the average planet mass, $M_{\mathrm{pl}}$. Our simulations suggest that $N \propto M_{\mathrm{HZ}}^{-0.1}$ or so. Indeed, a model with $M_{\mathrm{pl}} \propto M_{\mathrm{HZ}}^{1.1}$ provides a fit that is comparable to the one in Figure 2. However, for the remainder of the paper we assume that $M_{\mathrm{pl}} \propto M_{\mathrm{HZ}}$ (eq. [2]). The reason for this assumption has to do with the goals of the paper. We are attempting to constrain the locations in $M_{\star}-h-f Z$ parameter space that might harbor potentially habitable planets with $M_{\mathrm{pl}} \geq 0.3 M_{\oplus}$. To be conservative in our evaluations, we prefer to slightly overestimate, rather than underestimate, $M_{\mathrm{pl}}$. The difference between the two estimates is negligible for larger stellar masses, but is as much as $\sim 40 \%$ below $0.1 M_{\odot}$.

The planet mass decreases monotonically with stellar mass for all reasonable parameter values (eq. [2]; Fig. 3), with a scatter in the details of a given system based on the stochastic nature of the accretion process (e.g., Wetherill 1996). Only for very steep density profiles $(\alpha>2)$ or reversed disk mass scalings $(h<0)$ can the planet mass increase at lower stellar masses. These effects are the result of the strong dependence of the HZ's location on stellar luminosity and therefore on stellar mass. For a given value of $f, Z, \alpha$, and $h$, there exists a stellar mass limit below which the formation of a $>0.3 M_{\oplus}$ planet in the $\mathrm{HZ}$ is unlikely. For $\alpha=1$ and $h=1$, this limit ranges from $1 M_{\odot}$ for $f Z<0.3$ to $0.74 M_{\odot}$ for $f Z=1$ to $0.43 M_{\odot}$ for $f Z=5$. These limits clearly depend on the critical mass for habitability; for instance, the limit is $0.53 \mathrm{M}_{\odot}$ for the $f Z=1$ case if the critical habitable mass is $0.1 M_{\oplus}$. Recall that $f Z$ represents a scaling of the disk mass, i.e., the disk's relative mass $f$ times the relative abundance of solids, assumed to scale with the stellar metallicity $Z$.

Figure 4 shows the location in $M_{\star}-f Z$ space where planets $>0.3 M_{\oplus}$ can form in the HZ, assuming $\alpha=1$. Each curve corresponds to a given value of $h$; planets $>0.3 M_{\oplus}$ form above and

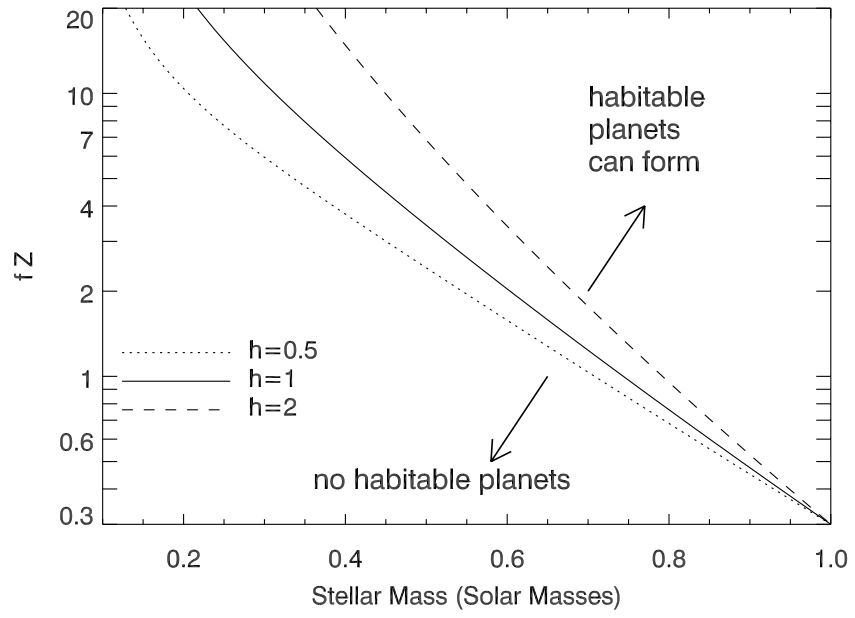

FIG. 4.- Regions of $M_{\star}-f Z$ space in which habitable planets more massive that $0.3 M_{\oplus}$ can form, assuming $\alpha=1$, for three different values of $h$. Planets larger than $0.3 M_{\oplus}$ can form above and to the right of each curve.

to the right of each curve. More massive or metal-rich disks can form habitable planets around lower mass stars. In addition, it is easier to form $>0.3 M_{\oplus}$ planets in the HZ for more centrally condensed disks, i.e., for larger values of $\alpha$ (not shown in Fig. 4). Given the large amount of variation in $f Z$ and other uncertainties, we do not consider these limits to be firm. However, given the large uncertainties and expected variation in $f$ and other parameters, we do not consider these limits to be meaningful except in a statistical sense. For an ensemble of disks, the fraction of $>0.3 M_{\oplus}$ planets that form decreases significantly for low-mass stars. A probabilistic version of the mass limit estimate is discussed further in $\S 4$.

\subsection{Formation Timescales and Planetary Water Contents}

Figure 5 shows the mean formation timescales for $\mathrm{HZ}$ planets in our simulations. Around $0.2 M_{\odot}$ stars, terrestrial planets in the $\mathrm{HZ}$ form in a few Myr. This increases to 20-50 Myr for Sun-like stars,

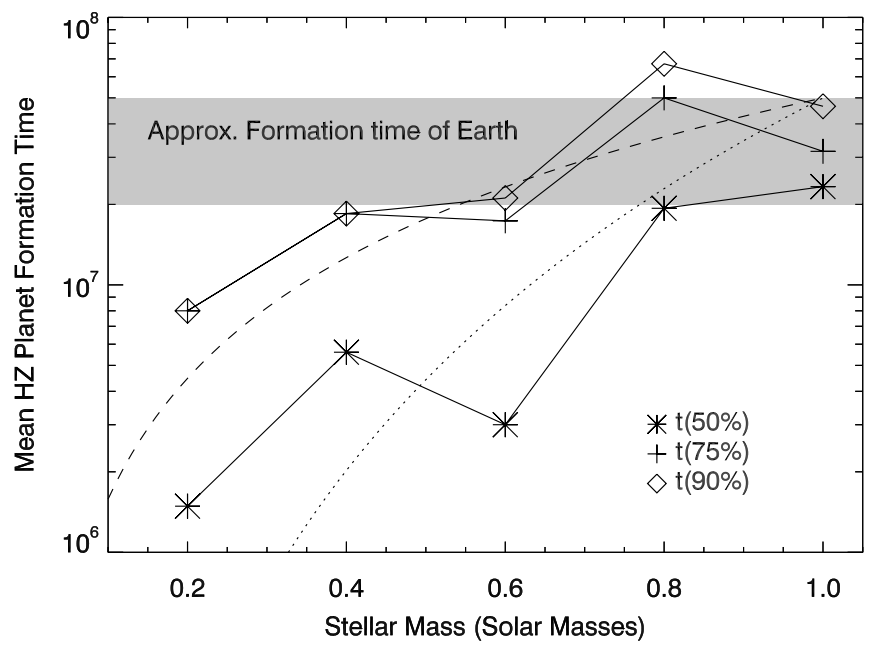

FIG. 5.-Formation times of HZ planets in our simulations. Different symbols correspond to the time for a planet to reach a fraction $(50 \%, 75 \%$, or $90 \%)$ of its final mass. Shaded regions show estimates for the formation time of the Earth, derived from $\mathrm{Hf} / \mathrm{W}$ isotopic measurements (e.g., Jacobsen 2005). The dotted line corresponds to a simple estimate from Safronov (1969), assuming that the formation time scales inversely with the product of the orbital frequency and the local surface density. The dashed line represents a different, simple model in which the formation time scales inversely as the product of the orbital velocity and the local surface density. Both estimates are referenced to $50 \mathrm{Myr}$ for $1 M_{\odot}$. 


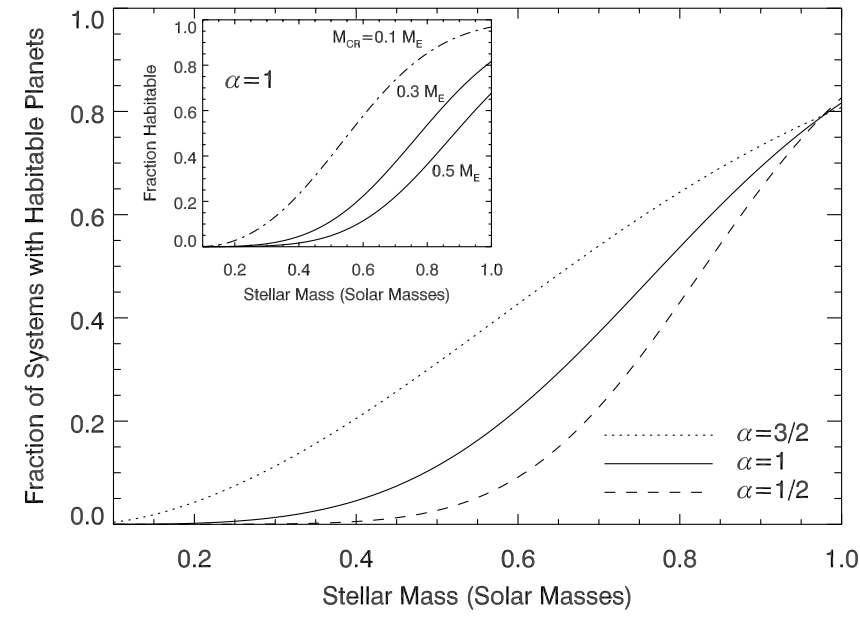

FIG. 6.- Fraction of stars able to form a $>0.3 M_{\oplus} \mathrm{HZ}$ planet as a function of stellar mass. We assume an $f Z$ distribution that is Gaussian, with a standard deviation of $0.5 \mathrm{dex}$ and a fixed value of $h=1$. The inset shows the effect of varying the limiting mass for habitability from 0.1 to $0.5 M_{\oplus}$, for $\alpha=h=1$.

consistent with estimates from $\mathrm{Hf} / \mathrm{W}$ isotopic measurements (e.g., Jacobsen 2005). The reason for the speedup in accretion times in the HZs of low-mass stars is due to a combination of the faster orbital speeds in the HZs of low-mass stars and the higher surface densities (albeit much lower total $\mathrm{HZ}$ masses). For instance, if we assume $L_{\star} \propto M_{\star}^{4}$ (a rough fit to Hillenbrand \& White 2004), then the location of the HZ, $r_{\mathrm{HZ}}$, scales with the stellar mass as $r_{\mathrm{HZ}} \propto$ $L_{\star}^{1 / 2} \propto M_{\star}^{2}$. The orbital speed in the HZ, $v_{\mathrm{HZ}}$, scales as $v_{\mathrm{HZ}} \propto$ $\left(M_{\star} / r_{\mathrm{HZ}}\right)^{1 / 2} \propto M_{\star}^{-1 / 2}$. The surface density in the HZ, $\Sigma_{\mathrm{HZ}}$, scales as $\Sigma_{\mathrm{HZ}} \propto r_{\mathrm{HZ}}^{-\alpha} M_{\star}^{h} \propto M_{\star}^{-2 \alpha+h} \propto M_{\star}^{-1}$ for our case of $\alpha=h=1$. So, using the very rough approximation that the growth time $t_{G}$ scales inversely with the product of the orbital frequency $\left(\sim v_{\mathrm{HZ}} / r_{\mathrm{HZ}}\right)$ and the local surface density (Safronov 1969) yields $t_{G} \propto M_{\star}^{7 / 2}$ for our case. This scaling is a very poor match to our simulations, yielding accretion timescales that are far too short for stellar masses below $0.6 M_{\odot}$ (Fig. 5, dotted line). This is because the final stage of planetary growth is dominated by isolated scattering events between embryos rather than accretion from a continuous medium of planetesimals. In fact, we note that a slightly different scaling, with $t_{G} \propto\left(\Sigma_{\mathrm{HZ}} v_{\mathrm{HZ}}\right)^{-1} \propto M_{\star}^{3 / 2}$, provides a good empirical fit to our simulated formation times (Fig. 5, dashed line). We suspect that this is simply because of the dynamics of this accretion regime, in which the relevant bodies are not on initially crossing orbits, and secular perturbations are required to excite eccentricities and thus to cause orbits to cross.

Lissauer (2007) argued that the formation time of HZ planets around low-mass stars is very short, a few hundred thousand years for a $1 / 3 M_{\odot}$ star. Our simulations confirm the trend that $\mathrm{HZ}$ planets form faster around low-mass stars. However, Figure 5 shows that these formation times do not scale nearly as strongly with stellar mass as indicated by Lissauer (he calculated $t_{G} \sim M_{\star}^{6.2}$ ). The reason for this discrepancy is that Lissauer required an Earthmass planet to form in the HZ, so to accomplish this he increased the disk mass by a large factor. Our models suggest that a factor of 20-30 increase is needed to form a $1 M_{\oplus}$ planet in the $\mathrm{HZ}$ of a $1 / 3 M_{\odot}$ star; as expected, that factor corresponds to the approximate difference in accretion times between our simulations and Lissauer's. However, the fraction of $1 / 3 M_{\odot}$ stars with disks massive enough to form a $1 M_{\oplus}$ planet in the $\mathrm{HZ}$ is less than $5 \%$, although that fraction increases to about $15 \%$ if $\alpha=3 / 2$ (see Fig. 6). In addition, recall that the much shallower empirical scaling $\left(t_{G} \propto\right.$ $M_{\star}^{3 / 2}$ for our case) provides a far better fit to our simulations than the simple derivation using the orbital frequency $\left(t_{G} \propto M_{\star}^{7 / 2}\right.$ for our case, based on Safronov [1969] and calculated in a fashion similar to Lissauer [2007]).

The mean water content of our HZ planets decreases drastically around low-mass stars. In fact, only 1 out of $19 \mathrm{HZ}$ planets formed from a star with $M_{\star}<0.6 M_{\odot}$ contained a significant amount of water, compared with two out of six HZ planets for $M_{\star}=0.8 M_{\odot}$ and two out of four for $M_{\star}=1 M_{\odot}$ (see Fig. 2). The reason for this trend-drier planets in the HZs of lower mass stars - is inefficient dynamical stirring in the low-mass disks found around low-mass stars, because more massive embryos are needed to increase eccentricities enough for significant radial mixing and therefore water delivery (Morbidelli et al. 2000; Raymond et al. 2004, 2007b). Lissauer (2007) argued that planets in the HZs of M stars would be deficient in volatiles for three reasons: (1) collision velocities are higher; (2) formation timescales are faster; and (3) pre-mainsequence evolution of the lowest mass $M$ stars is slow, such that the snow line and HZ move inward on a Myr to Gyr timescale (see Kennedy et al. 2006). We agree with point 1: collision velocities are proportional to the orbital speed, so the velocity of impactors at infinity (neglecting the escape speed) scales with stellar mass roughly as $M_{\star}^{-1 / 2}$ (see above). We also agree qualitatively with point 2: formation timescales are indeed likely to be shorter around low-mass stars by perhaps an order of magnitude compared with solar-mass stars (Fig. 5). Point 3 is more uncertain: the inward movement of the snow line could affect the water contents of HZ planets if the formation timescale is shorter than the snow line's drift timescale. In such a case, material in a given region may be too hot to contain water at early times, during accretion. After several Myr, that zone may drop below the threshold for water to condense. If, however, the planets are fully formed by this time, then water delivery is impossible. Note that this pre-main-sequence scenario applies only to very low-mass stars. Our results suggest that this is a moot point, as HZ planets around such very low-mass stars are unlikely to have wide enough feeding zones to accrete waterbearing material, even at late times.

Thus, we argue that terrestrial planets in the HZs of low-mass stars are likely to be dry, but for a different reason than Lissauer. Simply put, very little water-rich material is likely to impact such planets at all, because radial mixing is inefficient in the low-mass disks preferentially found around low-mass stars. The influence of additional gas or ice giant planets in the system is not expected to increase the water contents of $\mathrm{HZ}$ planets, at least not from the asteroidal source of water considered here (S. Raymond 2007, in preparation). However, migrating giant planets (not modeled here) can stir up eccentricities and induce the formation of very water-rich planets in their wake (Raymond et al. 2006a; Fogg \& Nelson 2007; Mandell et al. 2007). In addition, subsequent delivery of water from a cometary source is possible.

\subsection{Effects of Giant Planets}

An obvious criticism of this work is the lack of giant planets. Simulations have shown that giant planets play an important role in terrestrial planet formation (e.g., Wetherill 1996; Levison \& Agnor 2003). Giant planets on orbits exterior to the terrestrial region, such as Jupiter and Saturn, stir up the eccentricities of embryos from the outside in, via secular and resonant perturbations, while mutual scattering of embryos excites eccentricities from the inside out (e.g., Raymond et al. 2005b). Accretion is suppressed in the vicinity of the giant planet, because perturbations between embryos during their growth can scatter them into unstable regions such as strong giant planet resonances (Wetherill 1996). Indeed, the combined effects of embryo scattering and perturbations from Jupiter and Saturn are thought to be the cause of the depletion of 
the solar system's asteroid belt (Wetherill 1992; Chambers \& Wetherill 2001; Petit et al. 2001).

The net effect of giant planets is to increase the eccentricities of embryos during accretion. This can cause the width of the feeding zone of terrestrial planets to increase, causing planets to be somewhat more massive and less numerous than in the absence of giant planets (Levison \& Agnor 2003). This increase in planet mass can be as high as $\sim 30 \%$ (or planet masses can decreased), but occurs primarily for relatively weak perturbations, i.e., for less massive giant planets on relatively circular orbits (S. Raymond 2007 , in preparation). Even on circular orbits, external giant planets rarely enhance water delivery. Rather, their perturbations clear out external, water-rich material, leaving behind relatively dry terrestrial planets (S. Raymond 2007, in preparation). Indeed, terrestrial planets in systems with giant planets on eccentric orbits are likely to be dry (Chambers \& Cassen 2002; Raymond et al. 2004; Raymond 2006). Note that systems with close-in giant planets may also contain Earth-like planets, which should be very waterrich (Raymond et al. 2006a; Mandell et al. 2007).

To test the effects of giant planets, we performed eight additional simulations, including one giant planet on an exterior orbit. We chose the $0.6 M_{\odot}$ case for these simulations, to see whether giant planet perturbations could increase the mean terrestrial planet mass above $0.3 M_{\oplus}$. We used the same four sets of embryos described in Table 1 and included a giant planet on a circular orbit at $1.3 \mathrm{AU}$, corresponding roughly to the orbital distance with the same temperature (and therefore at a comparable dynamical separation from the $\mathrm{HZ}$ ) as Jupiter for a $0.6 M_{\odot}$ star. We ran two sets of simulations, one each for a Neptune- and a Jupiter-mass giant planet.

Planets that formed in the $\mathrm{HZ}$ of our $0.6 M_{\odot}$ simulations without giant planets had masses between 0.06 and $0.18 M_{\oplus}$, with a mean of $0.10 M_{\oplus}$. Systems with a Jupiter- (or Neptune-) mass giant planet formed, respectively, $\mathrm{HZ}$ planets with masses between $0.05[0.06]$ and $0.22[0.10] M_{\oplus}$, with a mean of $0.11[0.09] M_{\oplus}$, very close to the cases with no giant planets. None of the seven $\mathrm{HZ}$ planets in systems with no giant planets contained any waterrich material. Two out of $11 \mathrm{HZ}$ planets that formed in the eight giant planet systems contained a substantial amount of water. While this is only a small fraction of outcomes, it does show that giant planet stirring can, in some cases, promote water delivery. However, in the vast majority of situations, giant planets either hinder or have no effect on water delivery (S. Raymond 2007, in preparation). The formation timescales of $\mathrm{HZ}$ planets in giant planet simulations were comparable to, or even slightly longer than, those with no giant planets. In almost all cases, planets with or without giant planets reached $75 \%$ of their final masses within 10-20 Myr (see Fig. 5). We suspect that the reason giant planets do not accelerate accretion in this case is because more radial mixing is happening, such that accretion is somewhat less confined to a given annular zone. This effect appears to be small, as less than $20 \%$ of planets in the giant planet simulations contain water.

\section{DISCUSSION AND CONCLUSIONS}

Our analysis is based on three key assumptions: (1) terrestrial planets below a given mass are unlikely to sustain life on Gyr timescales - following Williams et al. (1997), we derive a limiting planet mass of roughly $0.3 M_{\oplus}$; (2) a protoplanetary disk can be well described by just a few parameters (see eq. [1]): the radial surface density slope $\alpha$, the relative disk mass in solids $f Z$, and the exponent characterizing the disk mass-stellar mass relationship, $h$; and (3) the typical planet mass in the habitable zone is proportional to the disk mass in that zone (see eq. [2]; Fig. 3).
We performed a suite of 20 dynamical simulations of the latestage growth of terrestrial planets that confirmed that this scaling does hold for a wide range of stellar masses. We assumed in our calculations that no giant planets were present. We tested that assumption for the $0.6 M_{\odot}$ case and found that the effects of exterior giant planets do not increase the masses or decrease the formation times of terrestrial planets, at least for the cases considered here.

Starting from these assumptions, we explored the range of parameters that allow planets more massive than $0.3 M_{\oplus}$ to form in the habitable zones (HZs) of their host stars. We found that any realistic combination of parameters led to a stellar mass-planet mass correlation such that $\mathrm{HZ}$ planet masses decrease with decreasing stellar mass (see Fig. 3). For a given set of parameters $(\alpha, h, f Z)$, one can derive a stellar mass below which the probability of a habitable planet forming in a disk with those parameters decreases to zero (see Fig. 4). For instance, for our fiducial parameters $(h=1, \alpha=1$, and $f=1.2$, chosen so that the simulations produce a mean planet mass of $1 M_{\oplus}$ for a $1 M_{\odot}$ star), this critical stellar mass is $0.7 M_{\odot}$. This would mean that no $\mathrm{M}$ stars and few $\mathrm{K}$ stars should have sustainably habitable planets. However, there are some caveats to this approach. First, for mainsequence stars, it is impossible to go back in time to learn the properties of their protoplanetary disks, most importantly, $f$ and $\alpha$. Second, for a given set of parameters, there still exists a range of potential outcomes because of the stochastic nature of the final stages of planetary growth (e.g., Wetherill 1996; Raymond et al. 2004; see Fig. 2). Third and most important, there is no universal set of parameters that applies for all disks. A given set of parameter values, corresponding to a specific disk or set of disks, has a corresponding planet mass distribution. For instance, the aforementioned $0.7 M_{\odot}$ limit decreases to $0.31 M_{\odot}$ for a more massive disk with $f Z=10$ and would decrease still further for $h<1$ or $\alpha>1$. Thus, our approach is viable only in a statistical sense, by combining likely outcomes from a distribution of disks with varying properties.

What are the values of the crucial parameters? We expect $h$ and $\alpha$ to be universal values characterizing the distributions of all disks, but $f$ and $Z$ to vary from disk to disk. As discussed above, there is considerable uncertainty in the value of $h$, which controls the disk mass-stellar mass scaling. If $h$ were larger than 2 or smaller than 0.5 , we expect that the trend would be apparent in Figure 3 in Scholz et al. (2006). Thus, a roughly linear disk mass-stellar mass relationship $(h=1)$ seems likely. A range of roughly 2 orders of magnitude in disk mass is observed around a given stellar mass (e.g., Eisner \& Carpenter 2003; Williams et al. 2005; Scholz et al. 2006), implying a corresponding range in $f$-values. Note, however, that there exists a large uncertainty in tying a given $f$-value to an absolute disk mass or surface density, because of both observational and theoretical uncertainties (e.g., Carpenter et al. 2005). There exists a range of 3-5 in metallicity $Z$ for thin-disk stars (Nordstrom et al. 2004), although the range for currently forming stars may be much smaller, only $\sim 20 \%$ (see Cartledge et al. 2006). Nearly all disk models and observations suggest that the surface density exponent $\alpha=0.5-1.5$ (e.g., Dullemond et al. 2007; Andrews \& Williams 2007). Our choice of $\alpha=1$ as a fiducial case was a compromise between these varying estimates.

Given the large amount of intrinsic variation in the disk mass and accretion outcome, as well as the uncertainty in $h$ and $\alpha$, we do not consider any stellar mass limits from Figure 4 to be firm. Rather, our primary result is a statistical observation that, in our framework, the fraction of disks capable of forming planets of a given mass in the habitable zone decreases around low-mass stars. We investigate a model with $f Z$ distributed as a Gaussian in 
$\log$ space, with a mean of $f Z=1$ and a standard deviation of 0.5 dex. For simplicity, all other parameters are held fixed, with $\alpha=1$ and $h=1$. Figure 6 shows that the fraction of systems that can form a $>0.3 M_{\oplus} \mathrm{HZ}$ planet decreases from $80 \%$ around solar-type stars to $42 \%$ around $0.7 M_{\odot}$ stars to $6 \%$ around $0.4 M_{\odot}$. stars to only $0.3 \%$ around $0.2 M_{\odot}$ stars. Disks with more centrally concentrated mass distributions (larger $\alpha$-values) have less difficulty forming massive planets in HZs very close to low-mass stars (e.g., Raymond et al. 2005b). For our chosen fiducial parameters, we estimate that only $25 \%$ of systems with $M_{\star}=0.6 M_{\odot}$ can form planets $>0.3 M_{\oplus}$, and only about $5 \%$ for $M_{\star}=0.4 M_{\odot}$. Disks with radial surface density profiles as flat as $r^{-1 / 2}$ have near-zero probability for stars with $M_{\star}<0.5 M_{\odot}$, while probabilities are 2-4 times larger for disks with $r^{-3 / 2}$ profiles. Reducing the required planetary mass for habitability to $0.1 M_{\odot}$ increases the probability by a factor of 2-4 (see below).

Our estimate of the minimum planet mass that can sustain enough tectonic activity for the period of time that we took as $5 \mathrm{Gyr}$ is also very uncertain. It is simple to show that if the massluminosity relation is of the form $L_{\star} \propto M_{\star}^{p}$, then the derived critical stellar mass $M_{\star, \mathrm{cr}}$ below which there should be few planets with

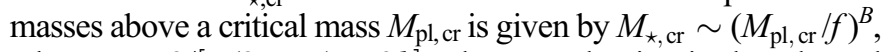
where $B=2 /[p(2-\alpha)+2 h]$. Thus, a reduction in the adopted critical mass for sustained habitability from 0.3 to $0.2 M_{\oplus}$ reduces the required value of disk mass normalization by the same factor, in order to obtain the same critical stellar mass. Alternatively, for a given chosen disk mass normalization, the derived $M_{\star, \text { cr }}$ varies with the assumed planet mass to a power that is between about 0.3 and 0.7 for most of the range of parameters described above. The inset of Figure 6 shows the effect of changing our $0.3 M_{\oplus}$ lower limit, retaining fixed values of $h=1$ and $\alpha=1$.

A system of three planets with minimum masses between 5 and $15 M_{\oplus}$ was recently discovered orbiting the $0.31 M_{\odot}$ star Gliese 581 (Bonfils et al. 2005; Udry et al. 2007). Figures 4 and 6 suggest that such a low-mass star is unlikely to form massive terrestrial planets. How can we reconcile this? We see two possible explanations: (1) the planets of Gl 581 formed in situ from a very massive disk, with $f Z \sim 30-50$, or (2) our assumption of in situ formation failed in this case: the planets of Gl 581 formed farther from the star (probably in the icy regions of a relatively massive disk, with $f Z$ of at least 5-10) and migrated inward (Goldreich \&
Tremaine 1980). If any of the Gl 581 planets were to transit the parent star, then it would be possible to derive a rough composition (rocky vs. icy; Valencia et al. 2007; Fortney et al. 2007; Sotin et al. 2007) and to distinguish between these two formation scenarios (Gaidos et al. 2007; Raymond et al. 2007a). Note that, even if the Gl 581 planets formed in situ, we expect that they would contain a substantial amount of water. Following the arguments made above, massive disks promote eccentricity growth and radial mixing such that planets in the $\mathrm{HZ}$ should accrete a large amount of water-rich material.

Even if $\mathrm{HZ}$ planets of sufficient mass can form around M stars, they face other challenges, including loss of atmosphere by intense stellar activity, coupled with their small distance to their parent stars, depending on the strength of the planetary magnetic field (Lammer et al. 2007), a snow line that takes perhaps a Gyr to get close enough to the HZ to produce sufficient water (Kennedy et al. 2006), or loss of volatiles due to larger impact speeds and faster formation times (Lissauer 2007; but see $\S 3.2$ and Fig. 5). However, these latter two points should not affect decisions concerning $\mathrm{M}$ star planet searches, since they apply only to the very lowest mass stars, $\sim 0.1-0.2 M_{\odot}$, whose apparent brightness and $\mathrm{HZ}$ angular separation are far too small for any planned search (Scalo et al. 2007). Unless the masses of disks are larger by a significant factor than is currently thought and/or the critical planet mass for habitability is considerably smaller, only a small fraction of accessible $\mathrm{M}$ star systems (with $M_{\star} \gtrsim 0.3-0.4 M_{\odot}$ ) should have habitable planets that remain habitable for billions of years.

This paper benefited from the referee's thoughtful comments and from discussions with Jim Kasting. This work was performed by the NASA Astrobiology Institute's Virtual Planetary Laboratory Lead Team, supported via NASA CAN-00-OSS-01. J. M. S. was supported by NASA Exobiology grant NNG04GK43G. S. N. R. was supported by an appointment to the NASA Postdoctoral Program at the University of Colorado's Center for Astrobiology, administered by Oak Ridge Associated Universities through a contract with NASA. Simulations were performed at Weber State University and JPL using CONDOR (see http://www.cs .wisc.edu/condor).
Abe, Y., Ohtani, E., Okuchi, T., Righter, K., \& Drake, M. 2000, in Origin of the Earth and the Moon, ed. K. Righter \& R. Canup (Tucson: Univ. Arizona Press), 413

Adams, F. C., Hollenbach, D., Laughlin, G., \& Gorti, U. 2004, ApJ, 611, 360

Alexander, R., \& Armitage, P. 2006, ApJ, 639, L83

Andre, P., \& Montmerle, T. 1994, ApJ, 420, 837

Andrews, S. M., \& Williams, J. P. 2005, ApJ, 631, 1134 2007, ApJ, 659, 705

Beckwith, S. V. W., Sargent, A. I., Chini, R. S., \& Guesten, R. 1990, AJ, 99, 924

Beer, M. E., King, A. R., Livio, M., \& Pringle, J. E. 2004, MNRAS, 354, 763 Beichman, C. A., et al. 2006, ApJ, 652, 1674

Bonfils, X., et al. 2005, A\&A, 443, L15

Butler, R. P., Johnson, J. A., Marcy, G. W., Wright, J. T., Vogt, S. S., \& Fischer, D. A. 2006, PASP, 118, 1685

Carpenter, J. M., Wolf, S., Schreyer, K., Launhardt, R., \& Henning, T. 2005, AJ, 129, 1049

Cartledge, S. I. B., Lauroesch, J. T., Meyer, D. M., \& Sofia, U. J. 2006, ApJ, 641,327

Chambers, J. E. 1999, MNRAS, 304, 793

2004, Earth Planet. Sci. Lett., 223, 241

Chambers, J. E., \& Cassen, P. 2002, Meteoritics Planet. Sci., 37, 1523

Chambers, J. E., \& Wetherill, G. W. 2001, Meteoritics Planet. Sci., 36, 381

Clarke, C. J., \& Pringle, J. E. 2006, MNRAS, 370, L10

Cyr, K. E., Sears, W. D., \& Lunine, J. I. 1998, Icarus, 135, 537

\section{EFERENCES}

D’Alessio, P., Canto, J., Calvet, N., \& Lizano, S. 1998, ApJ, 500, 411

Davis, S. S. 2005, ApJ, 627, L153

Dullemond, C. P., Hollenbach, D., Kamp, I., \& D’Alessio, P. 2007, in Protostars and Planets V, ed. B. Reipurth, D. Jewitt, \& K. Keil (Tucson: Univ. Arizona Press), 555

Dullemond, C. P., Natta, A., \& Testi, L. 2006, ApJ, 645, L69

Eisner, J. A., \& Carpenter, J. M. 2003, ApJ, 598, 1341 2006, ApJ, 641, 1162

Endl, M., Cochran, W. D., Kürster, M., Paulson, D. B., Wittenmyer, R. A., MacQueen, P. J., \& Tull, R. G. 2006, ApJ, 649, 436

Fischer, D. A., \& Valenti, J. 2005, ApJ, 622, 1102

Fogg, M. J., \& Nelson, R. P. 2007, A\&A, 461, 1195

Fortney, J. J., Marley, M. S., \& Barnes, J. W. 2007, ApJ, 659, 1661

Gaidos, E., Haghighpour, N., Agol, E., Latham, D., Raymond, S. N., \& Rayner, J. 2007, Science, in press

Garaud, P., \& Lin, D. N. C. 2007, ApJ, 654, 606

Gonzalez, G. 1997, MNRAS, 285, 403

Gould, A., et al. 2006, ApJ, 644, L37

Greaves, J. S., Fischer, D. A., \& Wyatt, M. C. 2006, MNRAS, 366, 283

Gregory, S. G., Jardine, M., Simpson, I., \& Donati, J.-F. 2006, MNRAS, 371, 999

Goldreich, P., \& Tremaine, S. 1980, ApJ, 241, 425

Hartmann, L., D’Alessio, P., Calvet, N., \& Muzerolle, J. 2006, ApJ, 648, 484

Hayashi, C. 1981, Prog. Theor. Phys. Suppl., 70, 35

Hillenbrand, L. A., \& White, R. J. 2004, ApJ, 604, 741 
Ida, S., \& Lin, D. N. C. 2005, ApJ, 626, 1045

Jacobsen, S. 2005, Annu. Rev. Earth Moon Planet. Sci., 33, 531

Kasting, J. F., Whitmire, D. P., \& Reynolds, R. T. 1993, Icarus, 101, 108

Kennedy, G. M., Kenyon, S. J., \& Bromley, B. C. 2006, ApJ, 650, L139

Kokubo, E., \& Ida, S. 1998, Icarus, 131, 171

Kokubo, E., Kominami, J., \& Ida, S. 2006, ApJ, 642, 1131

Lammer, H., et al. 2007, Astrobiology, 7, L185

Laughlin, G., Bodenheimer, P., \& Adams, F. C. 2004, ApJ, 612, L73

Lécuyer, C., Gillet, P., \& Robert, F. 1998, Chem. Geol., 145, 249

Levison, H. F., \& Agnor, C. 2003, AJ, 125, 2692

Levison, H. F., \& Duncan, M. J. 2000, AJ, 120, 2117

Lineweaver, C. H., \& Grether, D. 2003, ApJ, 598, 1350

Lissauer, J. J. 1999, Nature Suppl., 402, C11 2007, ApJ, 660, L149

Lodders, K., \& Fegley, B. 1998, The Planetary Scientist's Companion (New York: Oxford Univ. Press), 1998

Looney, L. W., Mundy, L. G., \& Welch, W. J. 2003, ApJ, 592, 255

Mandell, A. M., Raymond, S. N., \& Sigurdsson, S. 2007, ApJ, 660, 823

Morbidelli, A., Chambers, J., Lunine, J. I., Petit, J. M., Robert, F., Valsecchi, G. B.,

\& Cyr, K. E. 2000, Meteoritics Planet. Sci., 35, 1309

Moro-Martín, A., et al. 2007, ApJ, 658, 1312

Mundy, L. G., Looney, L. W., \& Welch, W. J. 2000, in Protostars and Planets IV, ed. V. Manning, A. P. Boss, \& S. S. Russell ( Tucson: Univ. Arizona Press), 355

Muzerolle, J., Hillenbrand, L., Calvet, N., Briceño, C., \& Hartmann, L. 2003, ApJ, 592, 266

Muzerollle, J., Luhman, K. L., Briceno, C., Hartmann, L., \& Calvet, N. 2005, ApJ, 625, 906

Natta, A., Testi, L., \& Randich, S. 2006, A\&A, 452, 245

Nordstrom, B., et al. 2004, A\&A, 418, 989

Padoan, P., Kritsuk, A., Norman, M. L., \& Nordlund, A. 2005, ApJ, 622, L61
Petit, J.-M., Morbidelli, A., \& Chambers, J. 2001, Icarus, 153, 338

Quinn, T. R., Tremaine, S., \& Duncan, M. 1991, AJ, 101, 2287

Rauch, K. P., \& Holman, M. 1999, AJ, 117, 1087

Raymond, S. N. 2006, ApJ, 643, L131

Raymond, S. N., Barnes, R., \& Mandell, A. M. 2007a, MNRAS, submitted Raymond, S. N., Mandell, A. M., \& Sigurdsson, S. 2006a, Science, 313, 1413 Raymond, S. N., Quinn, T., \& Lunine, J. I. 2004, Icarus, 168, 1 2005a, Icarus, 177, 256 2005b, ApJ, 632, 670

2006b, Icarus, 183, 265

2007b, Astrobiology, 7, 66

Reid, N., \& Hawley, S. L. 2000, New Light on Dark Stars: Red Dwarfs, Low Mass Stars, Brown Dwarfs, ed. N. Reid \& S. L. Hawley (New York: Springer) Safronov, V. S. 1969, Evolution of the Protoplanetary Cloud and Formation of the Earth and Planets (Moscow: Nauka) (English translation in NASA TTF677 [1972])

Santos, N. C., Israelian, G., \& Mayor, M. 2001, A\&A, 373, 1019

Scalo, J., et al. 2007, Astrobiology, 7, 85

Scholz, A., Jayawardhana, R., \& Wood, K. 2006, ApJ, 645, 1498

Sotin, C., Grasset, O., \& Mocquet, A. 2007, Icarus, in press

Tabachnik, S., \& Tremaine, S. 2002, MNRAS, 335, 151

Udry, S., et al. 2007, A\&A, 469, L43

Valencia, D., Sasselov, D. D., \& O'Connell, R. J. 2007, ApJ, 665, 1413

Walker, J. C. G., Hays, P. B., \& Kasting, J. F. 1981, J. Geophys. Res. 86, 9776

Ward, W. R., \& Hahn, J. M. 1995, ApJ, 440, L25

Weidenschilling, S. J. 1977, Ap\&SS, 51, 153

Wetherill, G. W. 1992, Icarus, 100, 307 1996, Icarus, 119, 219

Williams, D. M., Kasting, J. F., \& Wade, R. A. 1997, Nature, 385, 234

Williams, J. P., Andrews, S. M., \& Wilner, D. J. 2005, ApJ, 634, 495 